\title{
Influence du pré-mouillage sur les propriétés mécaniques d'un fil encollé
}

\author{
Nèjib Sejri ${ }^{1}$, Omar Harzallah ${ }^{2}$,a , Sami Ben Amar ${ }^{1}$, Sassi Ben Nasrallah ${ }^{1}$ \\ ET Pierre Viallier ${ }^{2}$ \\ 1 LEST, ENIM, Avenue Ibn El Jazzar, 5019 Monastir, Tunisie \\ 2 LPMT, ENSISA-UHA, 11 rue Alfred Werner, 68093 Mulhouse Cedex, France
}

Reçu le 8 juillet 2009, accepté le 21 juin 2010

\begin{abstract}
Résumé - L'encollage est un apprêt spécifique qui permet de conférer aux fils de chaîne une résistance mécanique leur permettant de supporter les contraintes du tissage sans détérioration. Cet apprêt doit en outre être éliminé à la fin du tissage avant l'ennoblissement et la pollution correspondante représente pratiquement $50 \%$ des rejets du teinturier. Depuis quelques années est apparue une technique qui consiste à imprégner d'eau les fils de chaîne avant de les foularder avec la solution de colle. On réduit ainsi la quantité de colle employée mais que se passe-t-il pour la mécanique des fils? Il est surprenant de constater que cette pré-imprégnation conduit avec moins de colle à des produits ayant un meilleur module de Young et un retour élastique plus important lors d'essais de fatigue. Nous avons ainsi lors d'examens microscopiques pu constater que ces résultats étaient dus à des points de colle plus «étalés ». Les essais de caractérisation physico-chimiques ont montré que cet étalement est dû à deux paramètres : on a une colle qui se dilue d'où une viscosité plus faible et surtout simultanément nous avons augmenté la tension de surface du fil en introduisant de l'eau entre les fibres d'où un meilleur mouillage. Un deuxième paramètre au moins aussi important est la diminution de la pilosité du fil encollé avec pré-mouillage.
\end{abstract}

Mots clés : Encollage / pré-mouillage / caractéristiques mécaniques / points de colle

\begin{abstract}
Influence of pre-wetting on the mechanical properties of a sized yarn. Sizing is a specific finish which makes it possible to confer on warp yarn a mechanical resistance enabling them to support the constraints of weaving without deterioration. This finish must moreover be eliminated at the end from weaving before the ennoblement and corresponding pollution accounts for practically $50 \%$ of the rejections of the dyer. For a few years a technique has appeared which consists in impregnating with water in the warp yarns before sizing them with the size solution. The quantity of size employed is thus reduced but what it happen for the mechanical properties of yarn? It is surprising to note that this pre impregnation leads with less size to products having a better Young modulus and a more important elastic return during fatigue tests. We thus during microscopic examinations could note that these results had with more "spread out" of size points. The physico-chemical tests of characterization showed that this spreading out is due to two parameters: there is a size which is diluted from where a lower viscosity and especially simultaneously we increased the surface tension of yarn by introducing water between fibres from where better wetting.
\end{abstract}

Key words: Sizing / pre-wetting / mechanical properties / size points

\section{Introduction}

L'encollage est un apprêt spécifique qui consiste à imprégner les fils de chaîne avec une colle hydrodispersible et à le sécher. Cet apprêt est indispensable pour conférer aux fils une résistance mécanique et pour réduire leur pilosité, de telle sorte que le tissage se passe sans problème [1].

\footnotetext{
${ }^{a}$ Auteur pour correspondance : o.harzallah@uha.fr
}

On mesure l'efficacité d'un encollage au nombre de casses de fils de chaînes pendant le tissage. Cette efficacité est liée à la quantité de colle déposée mais ce n'est pas le seul paramètre. En particulier, les fils de chaîne doivent conserver, malgré l'encollage, un module de flexion relativement faible pour être tissés (le tisserand parle de «souplesse » de fil). En outre, les fils doivent pouvoir passer à travers divers dispositifs mécaniques d'où l'intérêt de 


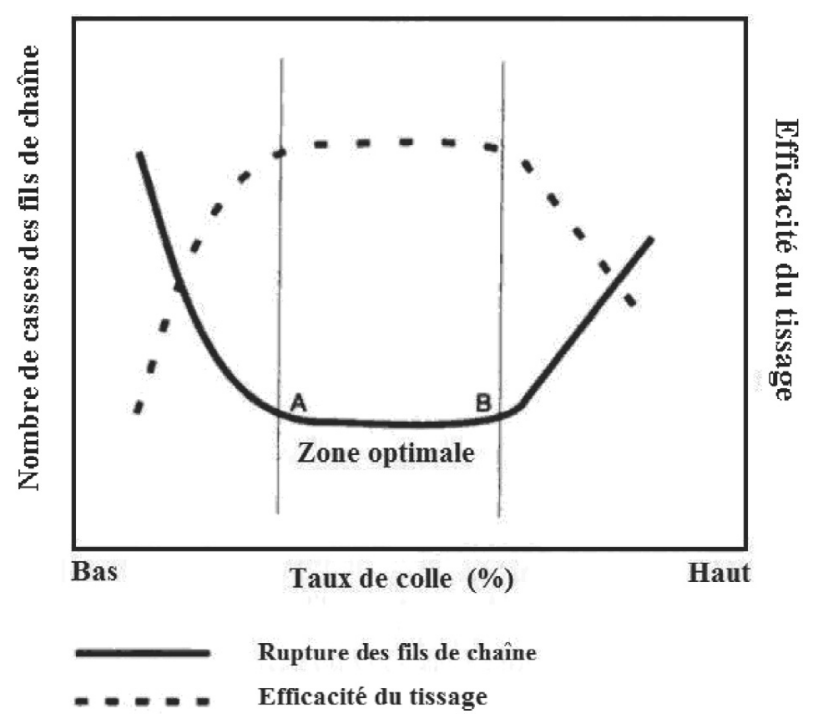

Fig. 1. Courbe typique de l'encollage-tissage [2]

réduire la pilosité du fil qui risque toujours d'« accrocher » et il y a également le risque d'accrochage entre fils voisins. Il faut donc introduire de la colle dans le fil, mais il existe une dose optimale comme l'ont montré Goswami et al. [2] (Fig. 1). Scholze et al. ainsi que Trauter et al. [3,4] ont tout spécialement étudié l'influence de la réduction de la pilosité du fil.

Pour notre part, nous avons vu apparaître dans l'industrie des encolleuses où, avant de faire l'imprégnation du fil par la colle, on l'imprégnait avec de l'eau chaude. A priori, avec moins de colle consommée, le tissage avait un aussi bon rendement. Or, dans la littérature, on trouve que l'encollage, outre son action sur la pilosité, fait croître le module des fils et surtout leur ténacité [5] et diminuer leur allongement à la rupture.

Cette étude a pour but de mieux comprendre les caractéristiques indispensables à un fil encollé et surtout l'influence du pré-mouillage des fils à l'eau chaude sur ces caractéristiques.

Nous avons donc réalisé des fils de coton que nous avons encollé, avec et sans pré-mouillage. La colle employée est un éther d'amidon.

\subsection{Influence de la technique d'encollage avec pré-mouillage sur les propriétés physiques d'un fil encollé}

\subsection{1 Évaluation du taux de colle}

Puisque la quantité de colle déposée a un effet sur le comportement mécanique d'un fil encollé lors du tissage, il est donc important de déterminer exactement cette quantité. Il existe plusieurs méthodes d'évaluation du taux de colle. La méthode la plus simple est de peser le fil avant et après encollage, ce qui est imprécis car nous n'avons pas le même taux d'humidité dans le fil avant et après encollage et dans le bain de colle chaud, le fil perd une partie de ses

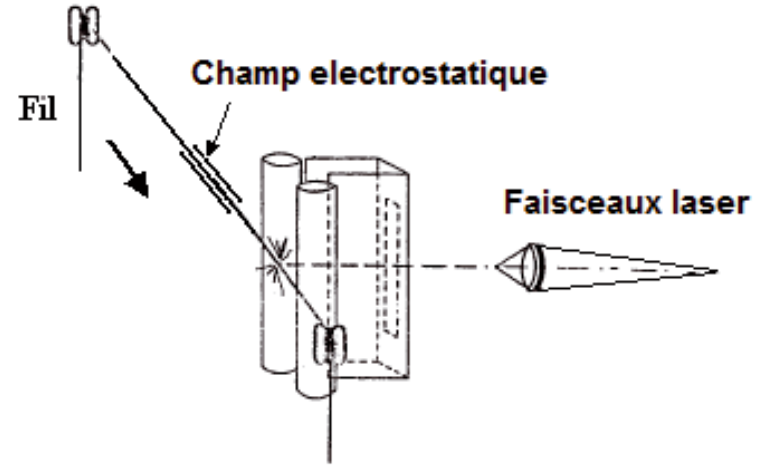

Fig. 2. Principe de fonctionnement du pilosimètre.

impuretés. La méthode la plus précise est le désencollage du fil [6]. C'est la technique utilisée dans cette étude. Les fils encollés sont séchés à $100{ }^{\circ} \mathrm{C}$, pesés, désencollés, séchés et pesés de nouveau. Le bain de désencollage contient de l'eau, un agent mouillant (Kieralon TX-BASF) et une enzyme (Enzymil Tex-Thor) pour hydrolyser les molécules d'amidon. Le fil utilisé pour cette étude est un fil de 42 tex, $100 \%$ coton « Open End $»$ produit par ValruptIndustrie.

Le tableau 1 regroupe les différents taux de colle pour les fils encollés avec et sans pré-mouillage. Le taux de colle est donné par la relation suivante :

$$
\begin{aligned}
\text { Taux de colle } \%= & 100 \times\left[\frac{\left(M_{\mathrm{fil}}\right. \text { encollé }}{M_{\mathrm{fil}} \text { désencollé }} M_{\mathrm{fil} \text { désencollé }}\right) \\
& \left.\left.-\frac{\left(M_{\mathrm{fil}}\right. \text { écru }}{M_{\text {fil désencollé }}}\right] M_{\mathrm{fil} \mathrm{désencollé}}\right)
\end{aligned}
$$

$\mathrm{Au}$ vu de ces résultats, nous pouvons déduire que le gain de colle en utilisant la nouvelle technique d'encollage est environ $16 \%$.

\section{2 Évaluation de la pilosité}

L'évaluation de la pilosité des fils a été réalisée à l'aide d'un pilosimètre (F098 Yarn Hairiness Tester Version 1.0 - SDL International, USTER). La figure 2 présente le principe de fonctionnement de ce pilosimètre.

Pour évaluer la pilosité nous avons choisi une longueur d'essai de $10 \mathrm{~m}$ de fil et une vitesse de déplacement de $50 \mathrm{~m} \cdot \mathrm{min}^{-1}$. La distance de détection de la fibre a été fixée à $3 \mathrm{~mm}$. On fait passer le fil dans un champ électrostatique pour redresser les poils, ensuite le fil est soumis à des faisceaux laser qui vont détecter tous les poils qui ont une longueur supérieure ou égale à la longueur de la pilosité libre choisie.

La figure 3 présente l'histogramme de la valeur moyenne du nombre de poils. $\mathrm{m}^{-1}$ pour les trois fils ainsi que l'intervalle de confiance correspondant. Elle montre que l'utilisation de la technique d'encollage avec prémouillage diminue fortement le nombre de poils.m ${ }^{-1}$. Nous constatons une réduction de $47 \%$ de la pilosité par rapport aux fils encollés sans pré-mouillage. En effet, l'eau 
Tableau 1. Gain de colle apporté par le pré-mouillage de la chaîne sur fil OE en production industrielle.

\begin{tabular}{ccc}
\hline \multirow{2}{*}{$\mathrm{N}^{\circ}$ essai } & \multicolumn{2}{c}{ Taux de colle $(\%)$} \\
\cline { 2 - 3 } & Fil encollé sans pré-mouillage & Fil encollé avec pré-mouillage \\
\hline 1 & 8,9 & 7,2 \\
2 & 8,5 & 7,4 \\
Valeur moyenne & 8,7 & 7,3 \\
\hline
\end{tabular}

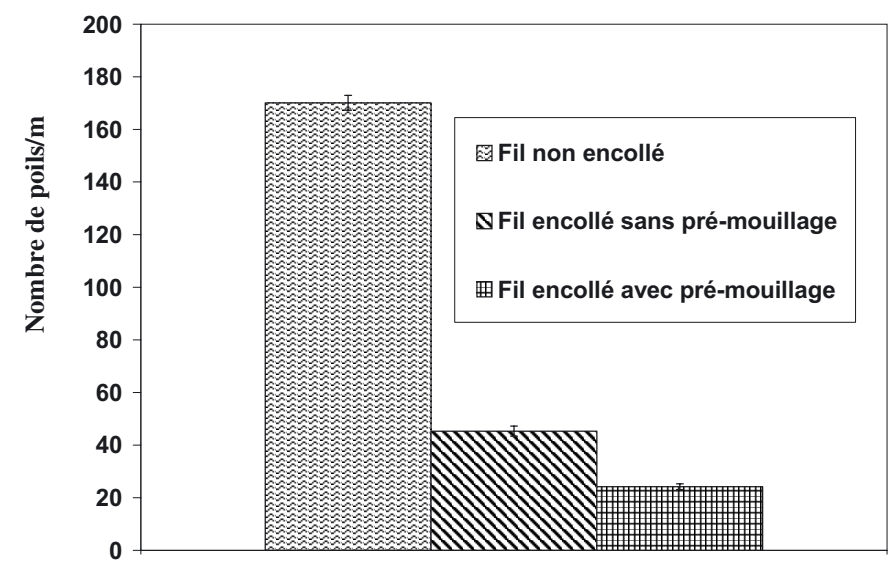

Fig. 3. Évaluation de la pilosité.

du premier exprimage rend les fibres plus souples d'où un meilleur écrasement lors de l'imprégnation dans la colle.

\section{Influence de la technique d'encollage avec pré-mouillage sur les propriétés mécaniques d'un fil encollé}

\section{1 Évaluation du module initial}

Nous avons réalisé des essais de traction et des essais de mise sous contrainte cycliques au moyen d'un dynamomètre de type (MTS 20) avec un capteur de $100 \mathrm{~N}$ à une température de $20^{\circ} \mathrm{C} \pm 2{ }^{\circ} \mathrm{C}$ et une humidité relative de $65 \% \pm 2 \%$. Conformément à la norme NFG07003, le temps de rupture est de $20 \pm 3 \mathrm{~s}$ et la longueur de l'éprouvette entre pinces est de $500 \mathrm{~mm}$ [7].

Pour chaque type de fil, une vingtaine d'éprouvettes ont été testées en traction. Le tableau 2 regroupe les valeurs de la ténacité et de l'allongement à la rupture des différents fils à savoir le fil non encollé, le fil encollé sans pré-mouillage et le fil encollé avec pré-mouillage. À partir de cet essai, nous avons pu suivre l'évolution de la force spécifique, $\mathrm{cN} /$ tex, en fonction de la déformation, \%. Les courbes portées sur la figure 4 représentent les courbes moyennes de traction des trois fils. La pente de la zone élastique des rhéogrammes obtenus permet d'accéder au module initial de chaque fil.

La figure 5 met en évidence l'évolution du module initial en fonction de la technique d'encollage. À partir des deux figures 4 et 5 , nous pouvons tirer les conclusions suivantes quant aux propriétés mécaniques des fils encollés avec les deux techniques d'encollage :

- La ténacité des fils encollés avec pré-mouillage est identique à celle des fils traités par l'encollage classique mais l'allongement à la rupture est plus faible. Ce résultat est logique : l'encollage ne supprime pas les défauts.

- Le module initial du fil encollé avec pré-mouillage est plus important que celui du fil encollé classiquement et le point d'inflexion de la partie élastique du rhéogramme est plus élevé. Cela veut dire que sur ce fil il sera plus difficile de dépasser la limite de la zone élastique et ceci est très souhaité au cours de l'opération de tissage pour éviter que tout arrêt entraîne un défaut.

Ce résultat est a priori surprenant : grâce au mouillage, nous mettons moins de colle dans le fil mais le module de Young croît. (Or, tout bon tisseur lorsqu'il a un problème avec une chaîne, augmente son taux de colle!).

- La limite élastique croît également.

\subsubsection{Analyse des propriétés de récupération des fils}

La théorie de l'élasticité concerne les déformations « élastiques » des solides, c'est-à-dire les déformations réversibles - généralement faibles. Lors du tissage, la meilleure nappe de fils de chaîne est celle qui a une bonne récupération élastique. La figure 6 [8] montre que dans le cadre d'un cycle d'hystérésis nous pouvons décomposer la déformation en deux parties : une partie élastique, qui est récupérée quand l'effort ou la déformation est supprimé(e) et une partie plastique ou permanente. Pour réaliser ce graphe, la force a été portée en fonction du déplacement de la traverse. Concernant notre étude, le comportement en extension cyclique cumulative a été étudié au moyen d'un dynamomètre de type MTS20. Ce comportement est une combinaison des effets de la récupération élastique, de la viscoélasticité et de la fatigue. Une éprouvette de $25 \mathrm{~cm}$ est placée entre les mors. Après que le fil ait subi une déformation de $10 \%$ de l'allongement à la rupture, par exemple, ce dernier revient à sa position initiale à la même vitesse. La traverse repart alors et le fil est étiré de nouveau jusqu'à $20 \%$ de l'allongement à la rupture. La procédure est, ensuite, répétée pour $30 \%$... jusqu'à sa rupture. Pour faciliter l'interprétation des résultats et éviter le chevauchement des cycles, la force a été tracée en fonction du temps multiplié par la vitesse de la traverse comme le montre la figure 7 . À partir de la courbe 
Tableau 2. Ténacité et déformation à la rupture des fils initiaux et encollés avec et sans pré-mouillage.

\begin{tabular}{|c|c|c|c|c|c|c|}
\hline & \multicolumn{2}{|c|}{ Fil non encollé } & \multicolumn{2}{|c|}{ Encollé sans pré-mouillage } & \multicolumn{2}{|c|}{ Encollé avec pré-mouillage } \\
\hline & $\begin{array}{c}\varepsilon \text { à la rupture } \\
(\%)\end{array}$ & $\begin{array}{l}\text { Ténacité } \\
(\mathrm{cN} / \text { tex })\end{array}$ & $\begin{array}{c}\varepsilon \text { à la rupture } \\
(\%)\end{array}$ & $\begin{array}{l}\text { Ténacité } \\
(\mathrm{cN} / \text { tex })\end{array}$ & $\begin{array}{c}\varepsilon \text { à la rupture } \\
(\%)\end{array}$ & $\begin{array}{l}\text { Ténacité } \\
\text { (cN/tex) }\end{array}$ \\
\hline Moyenne & 5,4 & 10 & 4,8 & 12,6 & 3,5 & 12,9 \\
\hline Écart type & 0,006 & 0,46 & 0,011 & 0,52 & 0,006 & 0,55 \\
\hline I. C. & 0,004 & 0,29 & 0,007 & 0,32 & 0,004 & 0,34 \\
\hline
\end{tabular}

I. C., Intervalle de confiance.

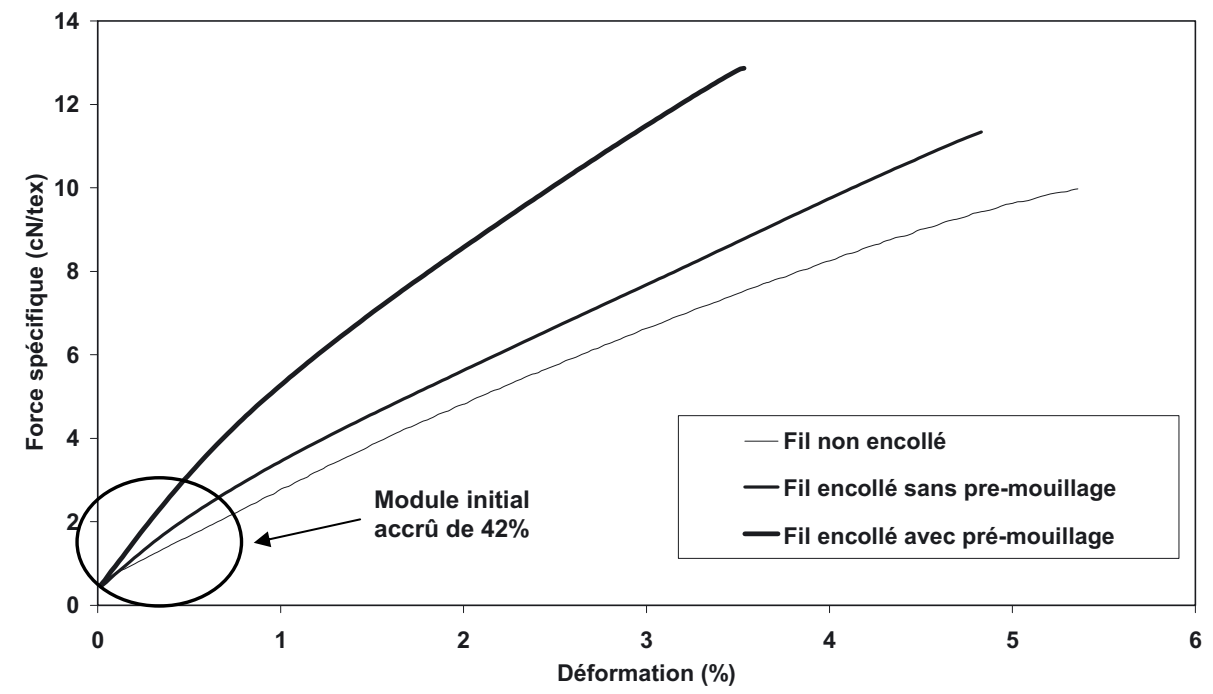

Fig. 4. Courbes force spécifique-déformation des trois types de fils.

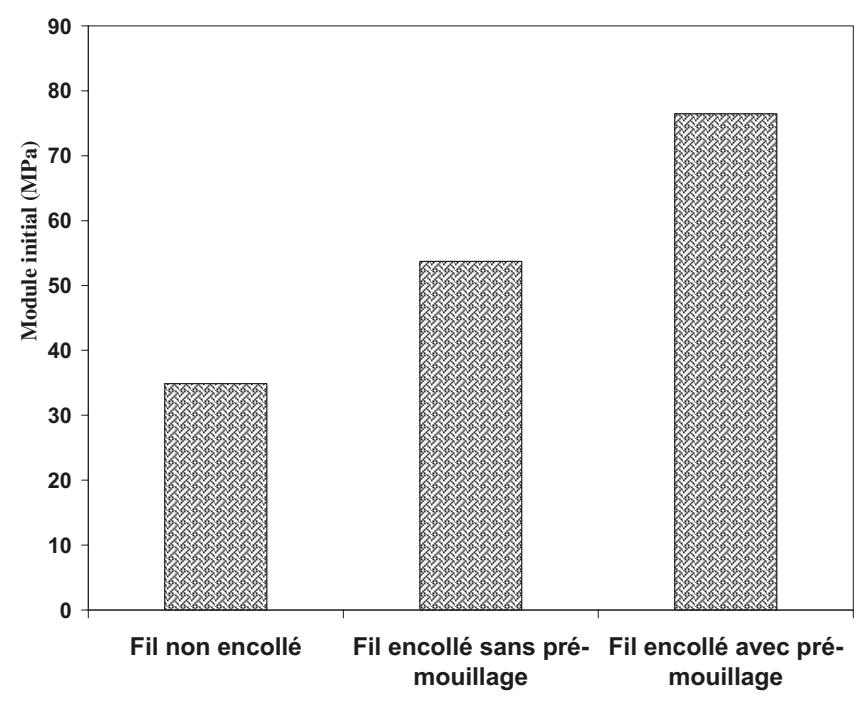

Fig. 5. Évolution du module initial en fonction de la technique d'encollage.

obtenue, la récupération immédiate ou élastique, différée et la déformation résiduelle sont extraites pour chaque cycle. La récupération élastique $(R 1 \%)$ qui est définie par le rapport entre l'allongement récupéré immédiatement $(A B)$ et l'allongement total $(O A)$ a pour expression :

$$
R 1 \%=100 \times A B / O A
$$

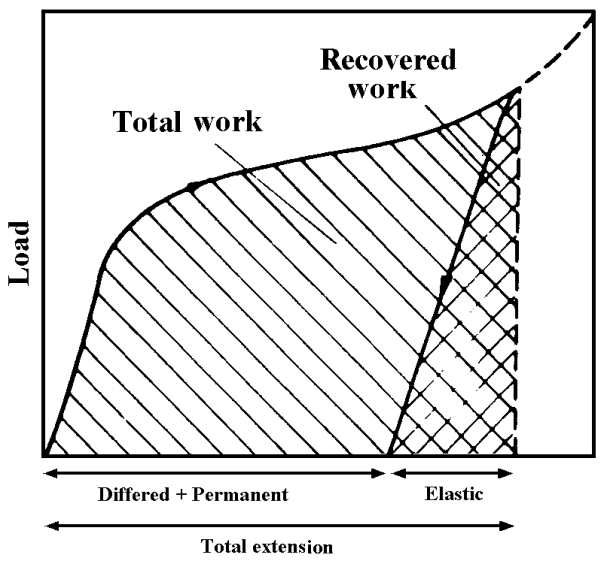

Fig. 6. Propriétés d'élasticité des fils [8].

La déformation résiduelle $(D \%)$ est donnée par le rapport entre l'allongement résiduel $\left(C O^{\prime}\right)$ et l'allongement total.

$$
D \%=100 \times C O^{\prime} / O A
$$

où $C$ et $O^{\prime}$ représentent, respectivement, le moment où la traverse revient à sa position initiale et le moment où le fil est étiré de nouveau.

D'après la figure 7 , nous pouvons constater que la distance $B C$ est supérieure à celle de l'allongement résiduel. 


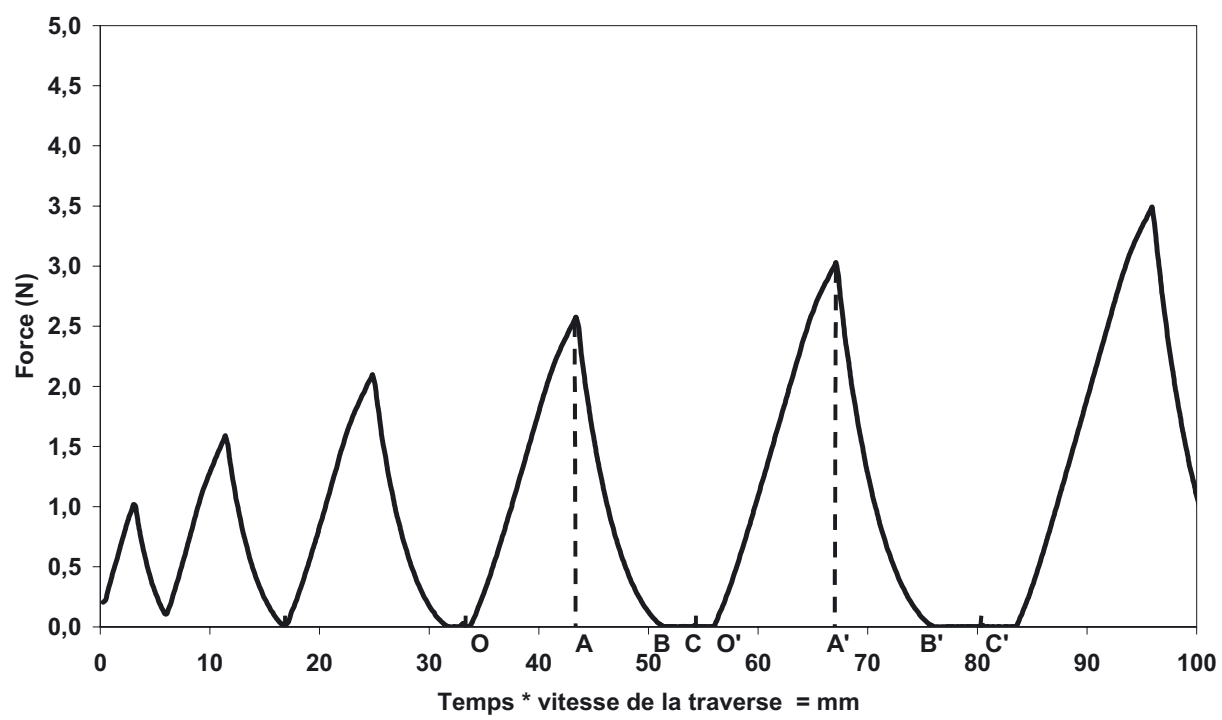

Fig. 7. Évolution de la force en fonction du temps multiplié par la vitesse de la traverse au cours d'un essai en extension cyclique cumulative.

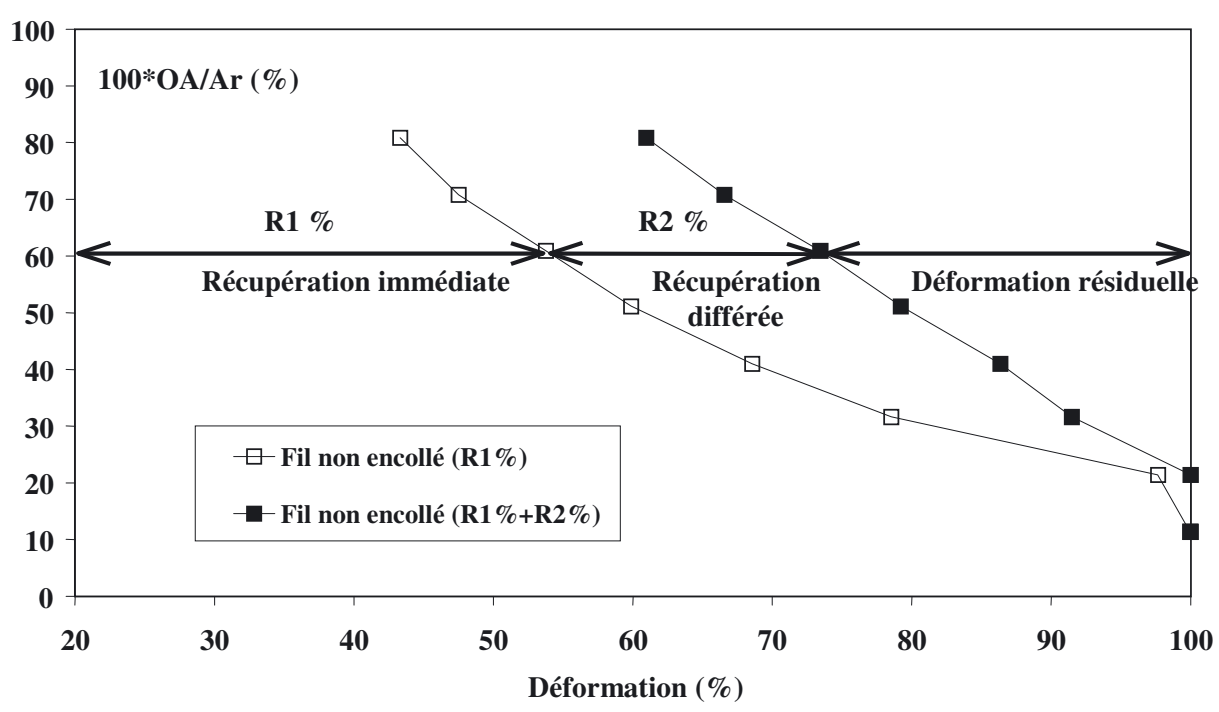

Fig. 8. Propriétés de viscoélasticité des fils non encollés.

De ce fait la récupération différée $(R 2 \%)$ peut être définie comme suit :

$$
R 2 \%=100 \times\left(B C-C O^{\prime}\right) / O A
$$

Pour chaque pourcentage de déformation imposée $100 \times$ $O A / A r(A r=$ allongement à la rupture), l'axe des $x$ est composé des deux zones de récupérations et de la déformation résiduelle comme le montre la figure 8 dans le cas d'un fil écru non encollé.

La figure 9 montre que le fil encollé avec pré-mouillage présente des récupérations immédiates plus importantes par rapport à un encollage classique et par conséquent il a une déformation résiduelle plus faible. Ce résultat confirme la croissance de la partie élastique.

\section{3 Étude de la diffusion de la colle et de sa morphologie}

Pour expliquer les améliorations des propriétés mécaniques des fils encollés avec pré-mouillage, nous avons étudié la cinétique de diffusion de la colle dans le tissu ainsi que sa morphologie dans le fil encollé à l'aide d'un microscope électronique à balayage (MEB) et ceci pour les deux techniques d'encollage.

\section{1 Étude de la morphologie de la colle dans le tissu}

Les figures 10 et 11 présentent les photos MEB des tissus encollés et séchés à $80^{\circ} \mathrm{C}$ pour deux concentrations de colle $\left(40\right.$ g.L ${ }^{-1}, 140$ g.L $\left.{ }^{-1}\right)$. 


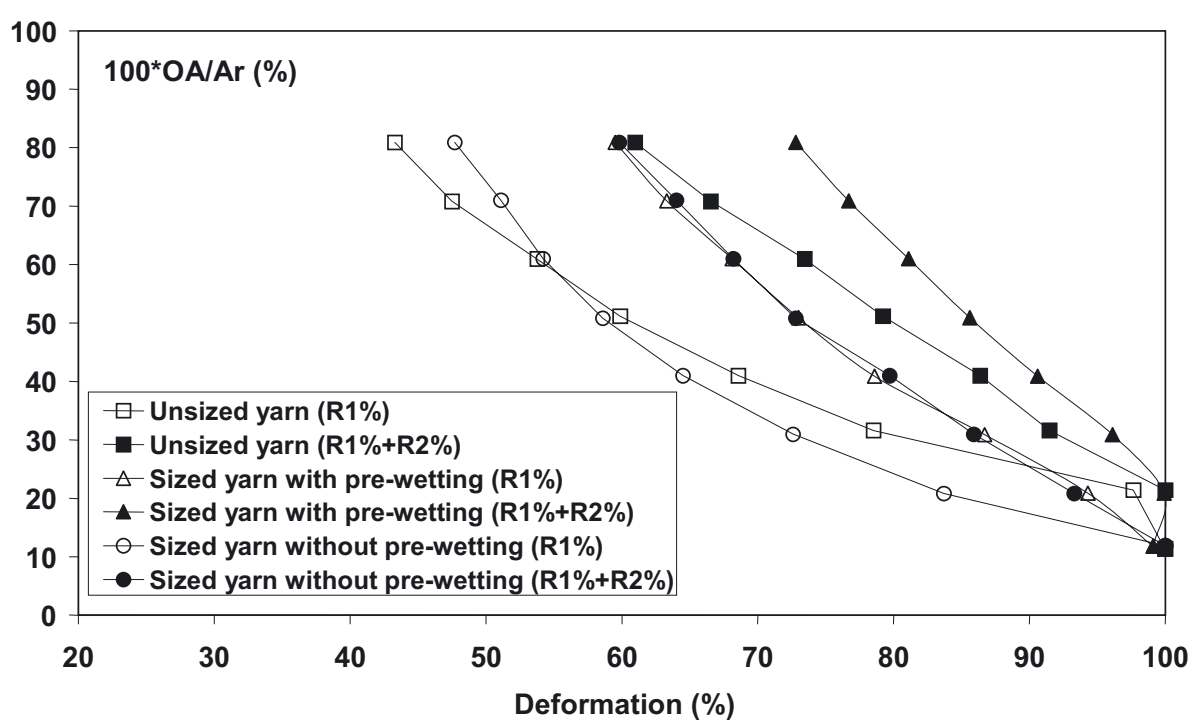

Fig. 9. Récupérations élastiques $(R 1 \%)$, différées $(R 2 \%)$ et déformations résiduelles pour les trois types de fils.

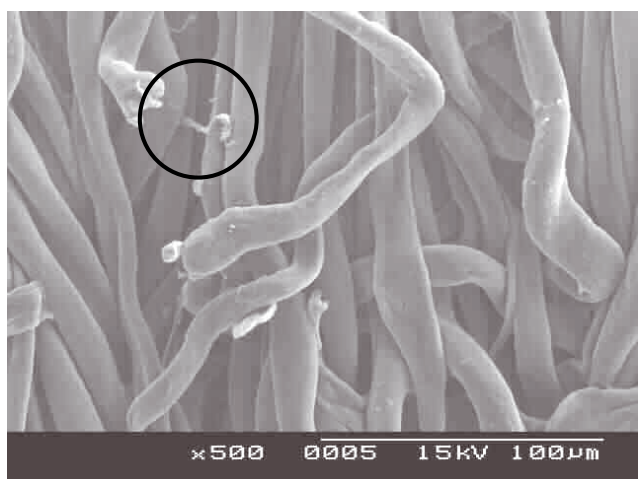

(a)

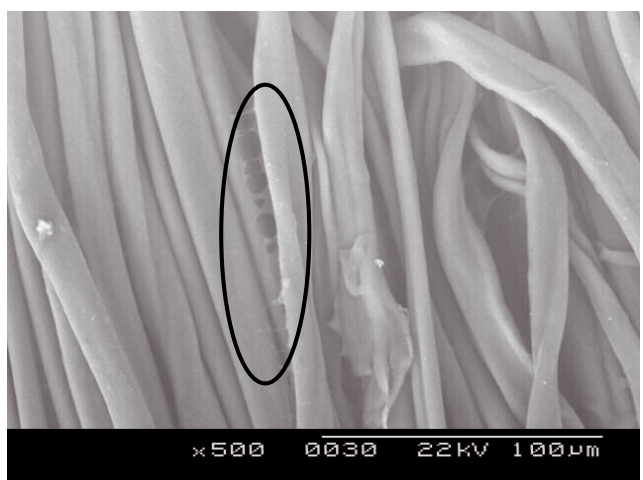

(b)

Fig. 10. Échantillon encollé, 40 g. $\mathrm{L}^{-1}$ de colle, $T s=80{ }^{\circ} \mathrm{C}$. (a) Encollage avec pré-mouillage. (b) Encollage sans prémouillage.

D'après les figures 10 et 11, nous remarquons que dans les tissus encollés avec les deux techniques, nous avons des points de colle dans les différents échantillons encollés et pas comme souvent dit un film de colle. La colle se

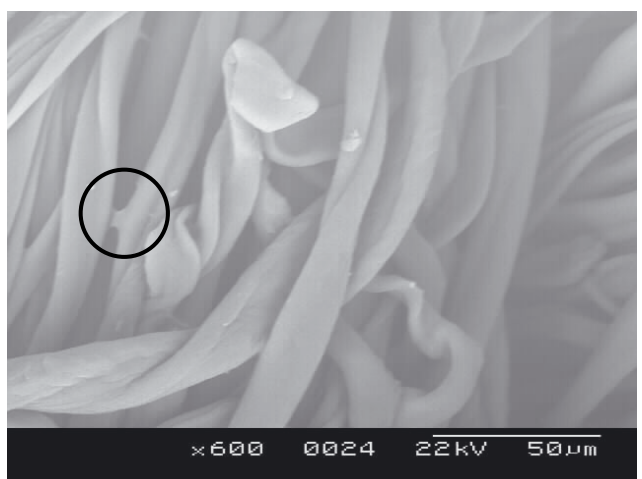

(a)

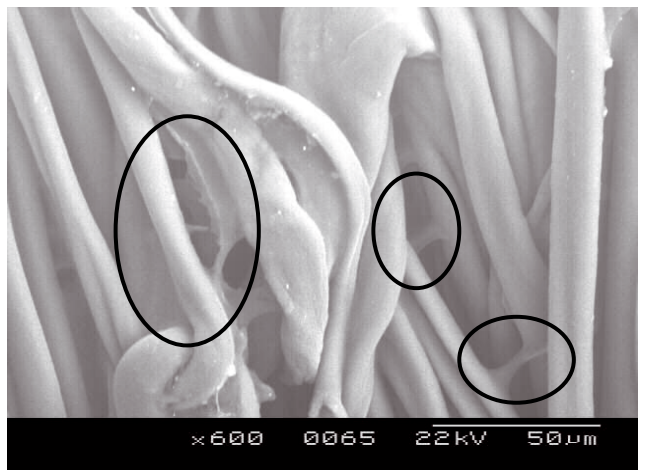

(b)

Fig. 11. Échantillon encollé, 140 g. $\mathrm{L}^{-1}$ de colle, $T s=80{ }^{\circ} \mathrm{C}$. (a) Encollage avec pré-mouillage. (b) Encollage sans prémouillage.

présente donc sous forme de ponts discontinus dans le cas de la nouvelle technologie, comme pour l'ancienne. Les points de colle semblent être plus épais dans le cas des échantillons encollés sans pré-mouillage. Ce résultat 


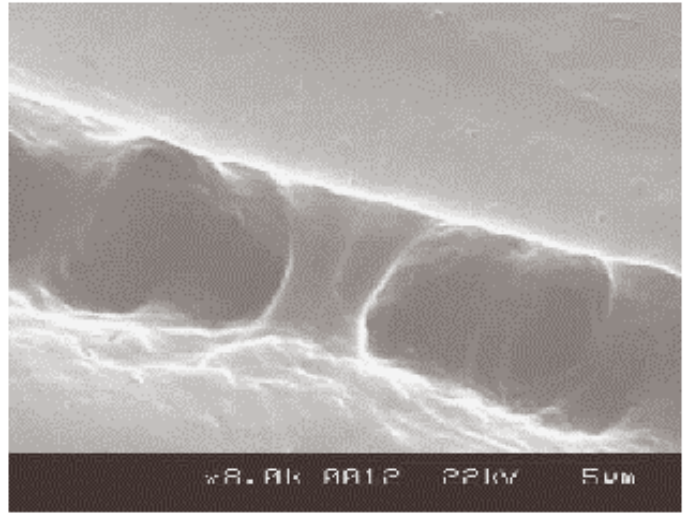

Fig. 12. Fil encollé sans pré-mouillage.

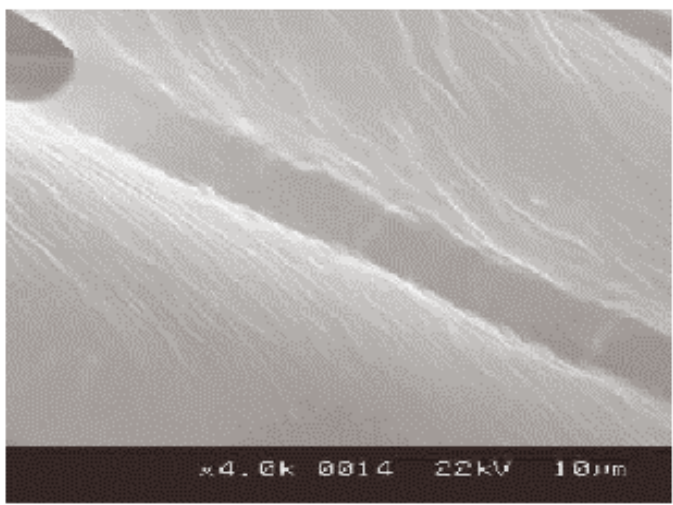

Fig. 13. Fil encollé avec pré-mouillage.

explique que le fil garde une grande souplesse, ce qui permet de l'enrouler et surtout de le tisser.

\section{2 Étude de la diffusion de la colle dans le fil encollé}

Nous avons utilisé les mêmes fils encollés lors de l'étude de l'influence du pré-mouillage sur les propriétés mécaniques pour réaliser les examens des points de colle. Des observations au MEB ont été réalisées pour étudier l'influence de cette nouvelle technologie sur la structure des points de colle ainsi que la pénétration de la colle dans le fil (Figs. 12 et 13).

D'après les figures 13 et 14 , nous remarquons que le fil encollé avec les deux techniques d'encollage n'est pas un matériau composite donc il reste assez souple pour être employé en tissage puisque la colle forme des points entre fibres. L'amélioration du comportement mécanique des fils de chaîne encollés selon cette nouvelle méthode de pré-mouillage peut s'expliquer par un meilleur étalement de la colle sur les faisceaux de fibres à la surface du fil par l'agent d'encollage. En effet, à la surface des fibres prémouillées la colle est légèrement diluée en contact avec l'eau. De ce fait, une diminution de la viscosité de la solution de colle est constatée. Ceci est accompagné par l'augmentation de la tension superficielle apparente du fil à cause de la présence de l'eau entre les capillaires, i.e.

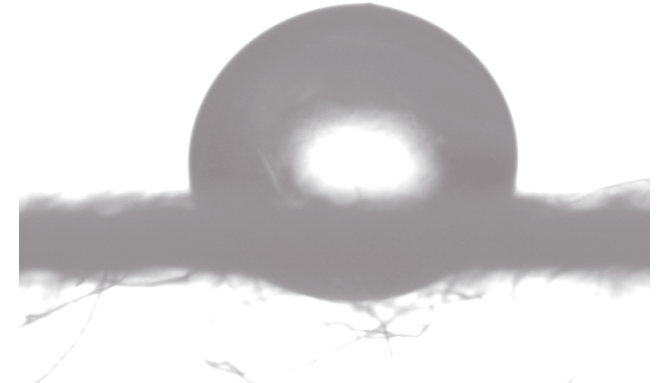

(a)

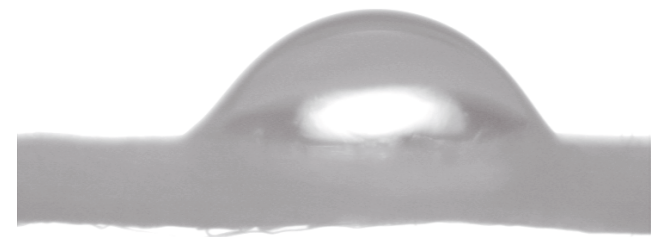

(b)

Fig. 14. Comportement d'une goutte de colle sur fil sec et mouillé. (a) Fil sec. (b) Fil mouillé.

entre les fibres, d'où une diminution de l'interface aircolle par le remplacement d'une fraction de l'air par l'eau (théorie de Cassie-Baxter) [9] lors de l'opération d'encollage. Ce phénomène est illustré par les photos 14a et 14b. On y voit le comportement d'une goutte de colle calibrée déposée sur fil sec et mouillé : sur ce dernier comme prévu, la colle s'étale le long du fil. C'est ce qu'on retrouve sur les figures 12 et 13 qui pourraient être reproduites à l'infini car cette différence d'aspect des points de colle est générale.

Du point de vue mécanique, on peut comparer le fil initial à un polyisoprène non vulcanisé; dès que l'on étire, les fibres vont glisser les unes par rapport aux autres, les seules forces s'opposant à ce mouvement étant les forces de frottement. La colle apporte une « vulcanisation $»$ mais, l'adhésion colle-fibre dépend évidemment de la taille de l'interface et des interactions colle-fibre à cette interface. Le pré-mouillage accroît la taille de l'interface. Ceci explique la croissance de l'élasticité.

\section{Conclusion}

Les résultats de cette étude, nous ont montré que le pré-mouillage apporte un gain de colle loin d'être négligeable (16\%) en conditions industrielles. Il semble, aussi, que cette diminution de teneur en colle ne dégrade pas les caractéristiques mécaniques du fil de chaîne vis-àvis du tissage. Au contraire le module initial croît de $42 \%$ par rapport à un fil encollé classiquement. Lors d'essais de fatigue nous avons pu montrer, également, que ce fil prémouillé présente des récupérations immédiates et différées accrues par rapport aux fils non pré-mouillé. Enfin, la mesure de la pilosité a permis de constater une chute de $47 \%$ par rapport à un fil encollé classiquement. 
Nous avons vu que l'amélioration des propriétés mécaniques et physiques, augmentation du module initial et diminution de la pilosité, peuvent être expliquées par une diminution de la viscosité de la solution de colle en contact avec l'eau et une augmentation de la tension superficielle à la surface des fils pré-mouillés, d'où un meilleur étalement de la colle. Ceci a été observé, aussi, à l'aide du microscope électronique à balayage. En effet, l'examen microscopique des points de colle semble montrer un meilleur étalement de ces points sur les fils prémouillés par rapport aux non pré-mouillés.

\section{Références}

[1] D. Nason, A nonaqueous method for sizing wool yarns: preliminary work, Textile Res. J. 58 (1988) 116-122

[2] B.C. Goswami, R.D. Anandjiwala, D.M. Hall, Textile Sizing, ISBN: 0824750535 Marcel Dekker (CRC Press), New York, 2004, pp. 243-247
[3] U. Scholze, J. Trauter, Hairiness- its effect on the weaving performance of sized warp yarns (part I), Textil Praxis Int. 10 (1993) 773-779

[4] J. Trauter, R. Vialon, Sizing of OE friction spun yarns, Textil Praxis Int. 8 (1990) 681-683

[5] S.D. Slauson, B. Millier, L. Rebenfeld, Physicochemical Properties of Sized Yarns: Part I: Initial Studies, Textile Res. J. 54 (1984) 655-664

[6] L.W.C. Miles, The Drying of textile Materials, Rev. Prog. Coloration 15 (1985) 21-24

[7] Essai des fils, Détermination de la force et de l'allongement de rupture par traction, méthode simplifiée, NFG 07-002, Paris : AFNOR, 1995

[8] W.E. Morton, J.W.S. Hearle, Physical Properties of Textile Fibres, The Textile Institute, Manchester, UK, 1993

[9] J. Drelich, J.D. Miller, A. Kumar, G.M. Whitesides, Wetting characteristics of liquid drops at heterogeneous surfaces, Colloids and surfaces 93 (1994) 1-13 